\title{
Vulnérabilité des cossettes issues de quelques cultivars d'igname à l'attaque de Dinoderus porcellus Lesne (Coleoptera: Bostrichidae) en conditions de laboratoire
}

\author{
Alexis ONZO ${ }^{1 *}$, Jeanine T. BIAOU ${ }^{1}$, Laura Y. LOKO ${ }^{2}$, Manuele TAMO $^{3}$ \\ et Alexandre DANSI ${ }^{2}$ \\ ${ }^{1}$ Faculté d'Agronomie, Département des Sciences et Techniques de Production Végétale, \\ Université de Parakou, B.P. 123, Parakou, Bénin. \\ ${ }^{2}$ Faculté des Sciences et Techniques de Dassa, Université Polytechnique d'Abomey, BP 14, Dassa, Bénin. \\ ${ }^{3}$ Biological Control Centre for Africa, International Institute of Tropical Agriculture, \\ 08 B.P. 0932, Cotonou, Benin. \\ *Auteur correspondant, E-mail: onzalex@yahoo.com
}

RÉSUMÉ

Dinoderus porcellus Lesne a été récemment identifié comme un véritable ravageur des stocks de cossettes d'igname en Afrique de l'Ouest. En conséquence, plusieurs méthodes de lutte ont été testées ou envisagées contre ce ravageur et comprennent, entre autres, l'utilisation des cultivars d'ignames résistants à l'insecte. Dans la présente étude, le comportement de quatre lots de cossettes issues des quatre cultivars d'igname suivants : Otoukpanan, Singor, Kprakpra et Portchahabim face à D. porcellus a été observé et comparé. Cette série d'études a porté sur: (1) la préférence de $D$. porcellus pour l'un ou l'autre des quatre cultivars; (2) la vitesse de développement et le taux d'émergence de $D$. porcellus sur ces différents cultivars d'igname, et (3) leur susceptibilité à travers les dégâts et l'effectif de la progéniture de $D$. porcellus enregistrés sur chacun d'eux. Nos résultats ont montré : que $D$. porcellus a une préférence marquée pour les cossettes de Otoukpanan suivi de Singor, Portchahabim et enfin Kprakpra; qu'il s'est développé plus rapidement sur Otoukpanan suivi de Singor, Portchahabim et Kprakpra; et qu'il a causé le plus de dégâts sur Otoukpanan sur lequel sa progéniture a été également la plus abondante. Portchahabim et Singor ont été les cultivars les moins attaqués du lot ; de plus, aucune progéniture n'a été enregistrée sur Portchahabim. Ainsi donc, les cultivars Singor et Portchahabim ont été les moins vulnérables aux attaques de $D$. porcellus et devraient être privilégiés dans la préparation des cossettes.

(C) 2014 International Formulae Group. All rights reserved.

Mots clés: Dioscorea sp., insectes des denrées stockées, préférence, résistance variétale, Bénin.

\section{INTRODUCTION}

L'igname (Dioscorea spp) de la famille des Dioscoréacées est une des plantes à racines et tubercules largement cultivées en Afrique de l'Ouest, et qui fournit à elle seule environ $95 \%$ de la production mondiale d'igname (FAO, 2012). Elle est cultivée généralement pour ses tubercules comestibles qui sont de bonnes sources de glucides, de vitamines et de minéraux, et fournissent plus de 200 calories par personne et par jour à plus de 60 millions de personnes (Olajumoke et al., 
2012). Le Bénin, quatrième pays producteur après le Nigéria, le Ghana et la Côte-d'Ivoire, a une production d'ignames estimée à 2.739.088 tonnes en 2012 (FAO, 2012). Plus de la moitié de la population béninoise l'a adoptée comme aliment de base et sa consommation par jour et par habitant est nettement plus élevée au Bénin (418 kcal) que dans les autres pays (FAO, 2012). Elle est d'ailleurs l'aliment préféré des populations du Centre et du Nord du pays, principale zone de production (Aboudou et Auriole, 2006), où elle est consommée à plein temps. Selon Baco et al. (2004), la production de l'igname fait désormais entièrement partie des mœurs et des traditions des populations au point qu'on peut parler d'une civilisation de l'igname. Plus qu'une culture de subsistance, l'igname est devenue une culture commerciale et joue un rôle déterminant tant du point de vue économique que socioculturel (Ondo et al., 2009). Aussi, a-t-elle été identifiée comme plante millénaire et culture d'avenir (Dégras, 1998). Les tubercules d'igname sont dégustés sous différentes formes: soit pilés, bouillis, rôtis (ragoûts), ou frits, et peuvent être également transformés en cossettes et par la suite en farine qui permet d'obtenir une pâte (communément appelée «Amala» en Yoruba) (Ayodeji et al., 2012 ; Oluwole et al., 2013).

Malgré ses fonctions économiques, alimentaires et culturelles, la production de l'igname demeure sérieusement freinée par de nombreux facteurs biotiques (ravageurs et maladies, etc.), et abiotiques (sols pauvres, changements climatiques, etc.). Aussi, la difficulté de conservation des tubercules frais occasionne des pertes post-récolte assez importantes (65-85\% du poids des tubercules), et une irrégularité de sa disponibilité tout au long de l'année (Ategbo et al., 1997 ; Babadjide et al., 2008). Cependant, depuis une vingtaine d'années, la production et la commercialisation des cossettes d'igname séchées au Bénin se sont très largement développées, tant et si bien que cette denrée est devenue aujourd'hui très importante dans l'alimentation des villes béninoises (Bricas et
Vernier, 2000). Ces cossettes qui s'obtiennent après pré-cuisson et séchage au soleil des tubercules épluchés (Vernier et al., 2000 ; Akissoé et al., 2007), permettent de limiter les pertes post-récolte et allongent la période de disponibilité du produit, renforçant ainsi la sécurité alimentaire (Aboudou et Auriole, 2006). Malheureusement, la productivité de cette activité est encore limitée par certaines contraintes, notamment, les attaques d'insectes sur les stocks (Ategbo et al., 1997; Demont et al., 2003 ; Loko et al., 2013).

En effet, les cossettes d'igname sont souvent sévèrement attaquées par un certain nombre d'insectes ravageurs qui peuvent réduire des stocks entiers en poudre en moins de quelques mois (Vernier et Dumont, 1995 ; Vernier et al., 2005). Parmi ces ravageurs, les insectes appartenant à l'ordre des coléoptères sont les plus abondants dans les stocks de cossettes d'igname (Adesuyi, 1967 ; Osuji, 1980 ; Loko et al., 2013). On y rencontre principalement : Dinoderus porcellus Lesne, Araecerus fasciculatus DeGeer, Sitophilus zeamais Motsch, Tribolium castaneum Herbst et Rhyzopertha dominica (Fabricius). Au Bénin, les espèces $D$. porcellus et $R$. dominica, tous appartenant à la famille Bostrichidae, se sont révélées les plus abondantes dans les stocks de cossettes d'igname (Kossou, 1987). De plus, les travaux effectués au Bénin (Loko et al., 2013), et au Nigéria (Osuji, 1980 ; Oni, 1995), ont montré de façon spécifique que $D$. porcellus est le principal ravageur des cossettes d'igname dans ces deux pays. En effet, D. porcellus est capable de réduire en poudre des stocks de cossettes d'ignames après seulement quatre à six mois de stockage (Osuji, 1980; Kossou, 1987 ; Oni, 1995 ; Loko et al., 2013).

Pour lutter contre ce ravageur, les paysans ont souvent recours aux insecticides chimiques. Malheureusement, cette méthode de gestion du ravageur a eu pour conséquence, aussi bien au Nigéria qu'au Bénin, de nombreux cas d'intoxication et de décès de familles entières suite à la consommation de cossettes d'igname contaminées par les substances utilisées pour leur stockage 
(Adedoyin et al., 2008; Adeleke, 2009). Il urge donc de trouver contre cet insecte des stocks une méthode de lutte préservant la santé humaine. Dans cette requête, l'utilisation de cultivars résistants est apparue comme une des meilleures options car très bénéfique pour les producteurs à faibles revenus. Heureusement, le Bénin dispose de cultivars d'igname présentant un certain degré de résistance aux attaques des insectes des stocks de cossettes (Dansi et al., 2003). On peut citer à cet effet, les cultivars Otoukpanan, Kprakpra, Singor, Portchahabim (Loko et al., 2013), qui méritent d'être testés.

La présente étude vise donc à déterminer au laboratoire les performances biologiques et démographiques de $D$. porcellus sur des cossettes issues de ces quatre différents cultivars d'igname précités en vue de caractériser leur degré de résistance à l'attaque du ravageur. L'objectif global de l'étude est donc de classifier les quatre cultivars d'igname par rapport à leur susceptibilité à l'attaque de D. porcellus.

\section{MATÉRIEL ET MÉTHODES}

\section{Site expérimental}

Les travaux ont été conduits dans les laboratoires de l'Institut International d'Agriculture Tropicale (IITA) à la station du Bénin. La station de l'IITA-Bénin est située dans la Commune d'Abomey-Calavi (Département de l'Atlantique), à environ 12 $\mathrm{km}$ au Nord-Ouest de Cotonou, à une altitude de $15 \mathrm{~m}$ au-dessus du niveau de la mer, à la latitude de $6^{\circ} 25^{\prime} \mathrm{N}$ et à la longitude de $2^{\circ} 19^{\prime} \mathrm{E}$.

\section{Matériel végétal}

Le matériel végétal est constitué de quatre cultivars d'igname appartenant au complexe D. cayenensis-D. rotundata; précisément au groupe variétal communément appelé "Kokoro" au Bénin. Le choix porté sur ces cultivars se traduit par le fait qu'à l'issue d'une évaluation participative avec les paysans, ces cultivars présentaient de bonnes caractéristiques agronomiques, culinaires et une résistance à l'attaque des insectes de stock (Loko et al., 2013). Il s'agit des cultivars:
Singor (récolté à Tchaourou: $8^{\circ} 52^{\prime} \mathrm{N}$; $2^{\circ} 36^{\prime} \mathrm{E}$ ); Kprakpra (récolté à Cobly : $10^{\circ} 29^{\prime} \mathrm{N} ; 1^{\circ} 01^{\prime} \mathrm{E}$ ) et Otoukpanan (récolté à Bantè : $\left.8^{\circ} 26^{\prime} \mathrm{N} ; 1^{\circ} 52^{\prime} \mathrm{E}\right)$.

Dans la présente étude, le cultivar Portchahabim (récolté à Ouaké : 9³9'N ; $1^{\circ} 23^{\prime} \mathrm{E}$ ), a été pris comme témoin en raison de sa susceptibilité déjà remarquée aux insectes de stock (Loko et al., 2013).

\section{Préparation des cossettes}

La préparation des cossettes d'igname a été faite suivant la méthode décrite par Babajide et al. (2006). En résumé, les tubercules d'igname ont d'abord été lavés avec de l'eau pour enlever la terre et autres matériels indésirables, puis pelés à l'aide d'un couteau et coupés en tranches. Les tranches d'igname ont été précuites à $50{ }^{\circ} \mathrm{C}$ pendant 2 heures dans de l'eau pour le blanchiment, puis laissées dans l'eau de pré-cuisson pendant 24 heures dans le but de les ramollir. Ensuite, les tranches ont été égouttées puis séchées à 60 ${ }^{\circ} \mathrm{C}$ à l'étuve pendant un minimum de 3 jours. Les tranches d'igname séchées ont enfin été conservées dans des sacs en polyéthylène à la température de $-4{ }^{\circ} \mathrm{C}$ tout au long de la période expérimentale (i.e. 3 mois environ).

\section{Matériel animal}

Les souches de D. porcellus utilisées ont été obtenues des cossettes d'igname du cultivar témoin (Portchahabim) achetées au marché. Toutes les cossettes ont été avant tout test, stérilisées dans une étuve à $100{ }^{\circ} \mathrm{C}$ pendant 2 heures afin de s'assurer qu'elles sont saines (Oni et Omoniyi, 2012). Le matériel d'élevage utilisé est constitué de boîtes carrées en plastique de $0,1 \mathrm{~m}$ x $0,1 \mathrm{~m}$ à la base et $0,08 \mathrm{~m}$ de hauteur. Ces boîtes ont été recouvertes par un couvercle en plastique criblé de trous de $1,5 * 1,5 \mathrm{~mm}$ de diamètre pour permettre l'aération du milieu de conservation et empêcher la sortie des insectes. Ces boîtes contenaient des cossettes de la variété Portchahabim et tous les stades de développement et sexes de $D$. porcellus. Les boîtes ont été ensuite disposées sur des 
étagères au laboratoire. Pour chaque expérimentation, seuls les insectes adultes âgés de 5-7 jours (i.e. ceux dont la couleur était bien foncée), ont été prélevés et utilisés (Isah et al., 2009). Durant l'expérimentation, la température et l'humidité relative au sein de l'enceinte d'étude ont fluctué respectivement entre 25-39 ${ }^{\circ} \mathrm{C}$ et $40-75 \%$. La durée d'éclairage a été de 12 heures.

\section{Méthodes}

Pour tester la vulnérabilité des cossettes issues des quatre cultivars d'igname aux attaques de D. porcellus, trois tests ont été effectués. Dans un premier temps, les insectes ont été mis dans une situation de choix pour déterminer si les cossettes exercent sur les insectes une attraction et donc que leur colonisation par D. porcellus n'était pas un fait de hasard. Dans un second test, un certain nombre d'œufs de D. porcellus ont été déposés sur les cossettes issues de chaque variété d'igname afin de déterminer les durées de développement des différents stades ainsi que le taux d'émergence des adultes par rapport à l'effectif initial. Enfin, dans le troisième test, nous avons quantifié les pertes occasionnées aux cossettes et déterminé comment la nourriture consommée a été convertie en progéniture.

\section{Expérience 1- Attractivité des cossettes issues des différents cultivars d'igname sur D. porcellus \\ Les essais ont été effectués au} laboratoire; le dispositif expérimental utilisé était celui de Babarinde et al. (2008). Au fond d'une boîte en plastique de $18 \mathrm{~cm}$ de diamètre, il a été collé à l'aide d'un adhésif (scotch à papier), un papier (papier A4) sur lequel il a été préalablement dessiné un cercle de $18 \mathrm{~cm}$ de diamètre. Ce cercle a été subdivisé en quatre parties égales. Sur chacune des subdivisions, une quantité de $5 \mathrm{~g}$ de cossettes d'igname issues de chaque cultivar à savoir : Singor, Kprakpra, Otoukpanan et Portchahabim a été déposée. Les cossettes ont été disposées à une distance de $9 \mathrm{~cm}$ du centre du cercle. Vingt-cinq adultes, tous sexes confondus, de $D$. porcellus préalablement affamés pendant $1 \mathrm{~h}$ ont été déposés au centre du cercle. Ainsi, les insectes pouvaient se déplacer librement vers les cossettes de leur choix (Isah et al., 2009). La boîte en plastique a été ensuite couverte avec un morceau de polyéthylène transparent qui assure l'aération $\mathrm{du}$ bocal tout en empêchant les insectes de s'échapper. Par séance, quatre boîtes en plastique (soit quatre répétitions) ont été utilisées. L'expérience a été reprise pendant 9 séances avec, à chaque fois, de nouveaux individus de $D$. porcellus et de nouvelles cossettes pour une variété d'igname donnée.

\section{Collecte et analyses statistiques des données}

Les données sur la distribution des insectes vers les cultivars et ceux restés sans choix ont été enregistrés après $24 \mathrm{~h}$ d'infestation (Babarinde et al., 2008). Les individus morts ou n'ayant opéré aucun choix ont été comptés. Sur l'ensemble des individus ayant choisi d'aller vers l'une ou l'autre des cossettes, les proportions de D. porcellus retrouvées sur chaque source (cultivar) de cossettes ont été calculées. Ces données ont ensuite été sujettes à une analyse de variance (Proc ANOVA), dans SAS (2008) afin de déterminer si les cultivars différaient entre eux dans l'attraction qu'ils exercent sur le ravageur. Lorsque l'analyse de variance révèle une différence significative, les proportions moyennes de $D$. porcellus attirées par chaque source (cultivar) de cossettes d'igname ont été séparées en utilisant le test de séparation multiple de Student-Newman-Keuls (SNK) dans SAS. Avant leur utilisation dans l'analyse de variance, les différentes proportions ont été transformées en utilisant l'arcsinus de leur racine-carrée (arsin (sqrt(Proportion)) afin d'homogénéiser leur variance. Les individus n'ayant effectué aucun choix n'ont pas été intégrés dans l'analyse statistique. 
Expérience 2- Influence des cossettes issues de différents cultivars d'igname sur la durée des différents stades de développement de $D$. porcellus

Les insectes utilisés pour cet essai ont été prélevés de l'élevage de masse et élevés sur les cossettes des différents cultivars d'igname en étude. Lors des travaux, il a été difficile de différencier le mâle de $D$. porcellus de la femelle (Cf. Schäfer et al., 2000). Ainsi donc, des adultes de $D$. porcellus, sans distinction de sexe, ont été introduits isolément dans une mini-boîte de Pétri $(2,5 \mathrm{~cm}$ de diamètre) et nourris avec de la farine de cossette issue de l'un des quatre cultivars d'igname en étude. Chaque insecte a été maintenu sur ce substrat alimentaire jusqu'à sa mort. Quotidiennement, les insectes ont été observés au microscope et ceux qui ont pondu des œufs - et qui, par conséquent, sont donc des femelles - ont été utilisés pour les tests. Lorsque les femelles introduites sur chaque cossette (i.e. différents cultivars d'igname) ont commencé par pondre, les nombres d'œufs pondus ont été enregistrés quotidiennement. Au total, 40 œufs ont été récoltés sur chacun des cultivars de cossettes d'ignames et transférés individuellement dans des mini-boîtes de Pétri numérotées de 1 à 40 et contenant de la farine du cultivar d'igname sur lequel la femelle ovipositante était initialement nourrie.

Les observations en rapport avec l'éclosion des œufs et le développement des stades juvéniles de $D$. porcellus ont été faites au microscope binoculaire (Wild M3B). Dès l'éclosion de chaque ouf, la larve a été maintenue sur le même substrat alimentaire, et sa survie a été suivie quotidiennement. Les dates de passage d'un stade de développement à un autre ont été enregistrées jusqu'à ce que l'individu atteigne le stade adulte ou qu'il meurt. Le nombre de pupes formées et d'adultes émergés ont alors été enregistrés. Les données collectées comprenaient les dates respectives : d'oviposition, d'éclosion de l'œuf, d'émergence de la larve, de formation de la pupe, d'émergence de l'insecte adulte ou de la mort de l'individu.

\section{Analyses statistiques}

Pour chaque cultivar d'igname, les proportions de $D$. porcellus ayant passé d'un stade de développement à un autre ont été calculées. Le taux d'émergence des adultes a été également déterminé. L'effet de la provenance des cossettes (i.e. différents cultivars d'igname) sur les durées de développement des différents stades juvéniles de $D$. porcellus a été déterminé en utilisant l'analyse de variance (Proc Anova) dans SAS (2008). Lorsque l'analyse de variance révèle une différence significative, les durées moyennes de développement de $D$. porcellus et le taux d'émergence des adultes sur les différentes cossettes issues des quatre cultivars d'igname ont été séparés en utilisant le test de séparation multiple de StudentNewman-Keuls (SNK) dans SAS (2008). Avant leur utilisation dans l'analyse de variance, les proportions ont été transformées en utilisant l'arcsinus de leur racine-carrée (arsin (sqrt(Proportion)), afin d'homogénéiser leur variance.

\section{Expérience 3- Evaluation des pertes de cossettes dues à $D$. porcellus et niveau de population du ravageur}

Ce test est basé sur la méthodologie utilisée par Isah et al. (2009). Ainsi, $50 \mathrm{~g}$ de cossette (tranches de cossette) d'igname de chaque cultivar ont été mis dans des boîtes carrées en plastique de $0,1 \mathrm{~m} \times 0,1 \mathrm{~m}$ à la base et $0,08 \mathrm{~m}$ de hauteur. Dans chaque boîte, les cossettes ont été infestées chacune avec 10 adultes de $D$. porcellus sans distinction de sexe. Chaque traitement a été répété 4 fois. Ces boîtes ont été recouvertes par un couvercle en plastique criblé de trous de $1,5 * 1,5 \mathrm{~mm}$ de diamètre pour permettre l'aération du milieu de conservation et empêcher la sortie des insectes. Ensuite, les boîtes ont été rangées au laboratoire et gardées pendant 12 semaines (Isah et al., 2009), période à la fin de laquelle les dégâts et pertes causés par $D$. porcellus ont été évalués. Le nombre total de $D$. porcellus vivants et de progéniture retrouvés dans chaque boîte ont été aussi enregistrés. Les proportions de 
cossettes consommées par le ravageur ont été estimées par l'évaluation du rapport poids perdu/poids initial.

L'effet de l'origine des cossettes (i.e. les différents cultivars d'igname) sur leur consommation par D. porcellus a été déterminé en utilisant l'analyse de variance (Proc Anova) dans SAS (2008). Lorsque l'analyse de variance révèle une différence significative, les proportions moyennes de cossettes consommées des différents cultivars d'igname ont été séparées en utilisant le test de séparation multiple de Student-NewmanKeuls (SNK), toujours dans SAS. Avant leur utilisation dans l'analyse de variance, les différentes proportions ont été transformées en utilisant l'arcsinus de leur racine-carrée ( $\operatorname{arsin}$ (sqrt(Proportion)), afin d'homogénéiser leur variance.

Les effectifs totaux de D. porcellus (tous stades confondus) retrouvés sur ou dans les cossettes des différents cultivars d'igname ont été comparés entre cultivars en utilisant l'analyse de variance (Proc Anova) dans SAS (2008). Lorsque l'analyse de variance révèle une différence significative, les effectifs de $D$. porcellus retrouvés par cultivar d'igname ont été séparés en utilisant le test de séparation multiple de Student-Newman-Keuls (SNK), toujours dans SAS.

\section{RÉSULTATS}

$\begin{aligned} & \text { Attractivité des cossettes issues des } \\ & \text { différents cultivars d'igname sur } D . \\ & \text { porcellus }\end{aligned}$

Des 900 insectes soumis aux tests (soit 25 adultes de $D$. porcellus testés pour 4 répétitions et repris 9 fois dans le temps), 61 étaient morts et n'ont donc pas été inclus dans les calculs subséquents. Sur les 839 individus vivants, en moyenne 641 insectes ont choisi d'aller sur l'une ou l'autre des quatre origines de cossettes mises à leur disposition (soit 76,4\%); tandis que les 198 restants (soit $23,6 \%$ ) n'avaient exprimé aucun choix. La distribution de $D$. porcellus sur les différentes origines des cossettes est représentée sur la Figure 1, qui montre que le flux le plus important de $D$. porcellus a été vers le cultivar
Otoukpanan avec en moyenne 24,62 $\pm 2,17 \%$ de l'effectif total soumis aux tests. Ensuite, vient le cultivar Singor avec en moyenne $18,69 \pm 1,45 \%$ de l'effectif total soumis aux tests, tandis que les cultivars Kprakpra et Portchahabim ont reçu les plus faibles flux de D. porcellus avec, respectivement, 16,68 \pm $1,58 \%$ et $16,72 \pm 1,58 \%$.

L'analyse de variance a révélé sur l'ensemble des neuf séries de test, une différence significative dans la distribution de $D$. porcellus sur les quatre origines de cossettes $(d l=3 ; \mathrm{F}=4,11 ; P=0,008)$. Le test de séparation multiple de SNK a révélé que la plus forte attirance a été vers le cultivar Otoukpanan suivi du cultivar Singor, tandis que l'attraction la plus faible a été vers les cultivars Kprakpra et Portchahabim, ces derniers n'étant pas significativement différents entre eux (Figure 1).

\section{Influence des variétés d'igname sur les durées des différents stades de développement de $D$. porcellus}

Stade œuf. La durée du stade œuf de D. porcellus se définit comme la période allant de la date de ponte à la date d'éclosion de l'œuf, c'est-à dire la date de l'émergence de la larve. Des 160 œufs isolés et suivis sur les quatre cultivars (soit 40 œufs sur chacun des 4 cultivars), au total 145 œufs ont éclos (soit 90,62\%). Les œufs, de couleur blanchâtre et oblongue, ont donné, après quelques jours, des larves de même couleur que les œufs. Les durées du stade œuf de D. porcellus sur chacun des cultivars d'igname sont présentées sur le Tableau 1, qui montre que la durée du stade œuf a été la plus courte sur le cultivar Otoukpanan avec en moyenne 4,00 \pm 0,24 jours, suivie du cultivar Singor avec 4,60 \pm 0,50 jours. Sur les cultivars Kprakpra et Portchahabim, la durée du stade œuf a été plus longue avec, respectivement, 5,00 $\pm 0,45$ jours et $5,38 \pm 0,53$ jours. L'analyse de variance a montré que les durées du stade œuf ne sont pas significativement différentes entre les différents cultivars d'igname utilisés pour préparer les cossettes $(d l=3 ; F=2,47 ; P=$ 0,077). 
Stade larvaire. La durée du stade larvaire de $D$. porcellus se définit comme l'intervalle de temps entre la date de l'émergence de la larve et la date de la formation de la pupe. Des 145 larves suivies sur les quatre cultivars, 46 pupes se sont formées (soit $31,72 \%$ des larves). Le Tableau 1 présente les durées du stade larvaire de $D$. porcellus sur les cossettes issues de chacun des cultivars d'igname testés. On y observe que le stade larvaire de $D$. porcellus a duré en moyenne $52,35 \pm 2,13$ jours sur le cultivar Otoukpanan, 63,80 \pm 4,05 jours sur le cultivar Singor, 73,00 $\pm 6,51$ jours sur le cultivar Kprakpra et 75,23 \pm 2,27 jours sur le cultivar Portchahabim. L'analyse de variance a révélé une différence significative entre les durées du stade larvaire de $D$. porcellus sur les différents cultivars d'igname utilisés pour fabriquer les cossettes $(d l=3 ; F=14,03 ; P=0,0001)$. Le test de séparation multiple de StudentNewman-Keuls (SNK) a révélé que le stade larvaire a été significativement plus court sur le cultivar Otoukpanan que sur les autres cultivars (Singor, Kprakpra et Portchahabim) qui n'ont présenté aucune différence significative entre eux.

Stade nymphal. Quelques jours (voire semaines) après la formation de la larve, une métamorphose se produisit, débouchant sur la formation d'un cocon dans lequel se trouve enfermée la nymphe (Tableau 1). La jeune nymphe ainsi formée, est de couleur blanchâtre et a déjà pris la forme de $D$. porcellus adulte. La durée du stade nymphal de $D$. porcellus se définit comme l'intervalle de temps entre la date de la formation du cocon et la date d'émergence de l'adulte. Des 46 nymphes suivies sur l'ensemble des cossettes provenant des quatre cultivars d'igname, 42 adultes ont émergé (soit 91,30\% du total des nymphes formées). Le Tableau 1 présente les durées du stade nymphal de $D$. porcellus sur chaque source de cossettes d'igname testées. La durée du stade nymphal de $D$. porcellus a été en moyenne 4,30 $\pm 0,40$ jours sur le cultivar Portchahabim, 4,59 \pm 0,45 jours sur le cultivar Otoukpanan, 6,40 \pm
1,69 jours sur le cultivar Singor et 10,83 \pm 6,25 jours sur le cultivar Kprakpra. Les durées de développement les plus longues ont été enregistrées sur le cultivar Kprakpra, suivi du cultivar Singor. Cependant, l'analyse de variance n'a montré aucune différence significative entre les durées du stade nymphal de $D$. porcellus, sur les cossettes issues des différents cultivars d'igname $(d l=$ $3 ; F=1,33 ; P=0,278)$.

$\mathrm{Au}$ total, sur un échantillon de 160 (40*4) œufs suivis, 145 ont éclos en donnant des larves (soit 90,62\%) et de ceux-ci, 46 ont formé des nymphes (soit $31,72 \%$ des larves) et des 46 nymphes formées, 42 sont parvenues au stade adulte (soit $91,30 \%$ du total des nymphes).

\section{Durée totale de développement et longévité des adultes}

Les durées totales de développement de D. porcellus (i.e. de l'œuf à l'adulte) par cultivar (Tableau 1) montrent que sur le cultivar Otoukpanan, la durée totale de développement de $D$. porcellus a été de 60,94 $\pm 2,20$ jours. Elle a été de 74,80 $\pm 5,20$ jours sur le cultivar Singor, de 84,92 $\pm 2,16$ jours sur le cultivar Portchahabim et de 88,33 \pm 7,73 jours sur le cultivar Kprakpra. L'analyse de variance a révélé une différence significative entre les durées de développement de $D$. porcellus sur les différents cultivars ayant produit les cossettes $(d l=3 ; F=15,84 ; P=0,0001)$. Le test de séparation multiple de Student-NewmanKeuls (SNK) a montré que le cultivar sur lequel le développement a été le plus rapide est Otoukpanan, alors que les trois autres cultivars à savoir Singor, Portchahabim et Kprakpra n'ont présenté aucune différence significative entre eux.

La longévité des adultes sur les différents cultivars (Tableau 1), fait remarquer que les adultes de $D$. porcellus ont vécu en moyenne $69,17 \pm 12,68$ jours sur le cultivar Kprakpra, 43,80 $\pm 6,97$ jours sur Singor, 38,54 \pm 4,80 jours sur Portchahabim et 37,59 \pm 4,58 jours sur Otoukpanan. L'analyse de variance a montré que la longévité des adultes 
de D. porcellus n'était, cependant, pas significativement différente entre les différents cultivars d'igname ayant servi à faire les cossettes $(d l=3 ; F=2,58 ; P=$ $0,068)$.

\section{Eclosion des oufs de $D$. porcellus sur les} différents cultivars

Il est à noter que des 40 œufs isolés et déposés sur chacun des substrats (cossettes provenant des différents cultivars d'igname), il a été observé que sur la variété Portchahabim, 38 des 40 œufs ont éclos, soit un taux d'éclosion de $95 \%$, et que $32,5 \%$ ont survécus pour donner des adultes (Figure 2). Sur les variétés Otoukpanan et Singor, 37 des 40 œufs ont éclos, soit un taux d'éclosion de $92,5 \%$; mais le nombre de $D$. porcellus émergés a été de 17 sur Otoukpanan (soit $42,5 \%$ ) et de 5 insectes, soit $12,5 \%$ sur la variété Singor (Figure 2). Sur la variété Kprakpra, 33 œufs sur les 40 ont éclos, soit un taux d'éclosion de $82,5 \%$ et 7 adultes de $D$. porcellus ont émergé, soit 17,5\% (Figure 2).

\section{Evaluation des pertes dues à $D$. porcellus et} niveau de population du ravageur

Les pertes causées par $D$. porcellus sur les cossettes issues des différents cultivars montrent que les cultivars Portchahabim et Singor ont été les moins attaquées avec des pertes similaires, soit $1,33 \pm 0,7 \%$ du poids initial des cossettes. Les variétés Otoukpanan et Kprakpra ont été les plus consommées par les insectes et les dégâts causés ont été quantitativement similaires avec des pertes moyennes de poids de $2,0 \pm 0,0 \%$ et $2,0 \pm$ $1,2 \%$, respectivement. L'analyse de variance a révélé qu'il n'y a aucune différence significative entre les pertes induites par $D$. porcellus sur les différents cultivars $(d l=3$; $F=0,25 ; P=0,85)$.

Après 12 semaines, le nombre d'insectes adultes vivant retrouvés sur les 10 individus constituant la population initiale par cultivar a été en moyenne de 8,0 $\pm 0,57$ sur Otoukpanan, 5,33 \pm 2,02 sur Kprakpra, 4,33 \pm 0,33 sur Singor et $3,67 \pm 0,33$ sur Portchahabim (Tableau 2). L'analyse de variance (Tableau 2) n'a révélé aucune différence significative entre le nombre de $D$. porcellus adultes survivant sur les quatre différents cultivars testés $(d l=3 ; F=2,42$; $P=0,14)$.

L'effectif de la progéniture (œufs + larves) de $D$. porcellus sur les différents cultivars d'ignames au cours des 12 semaines a été de 35,0 $\pm 11,13$ sur Otoukpanan, 3,67 \pm 3,67 sur Singor, 0,33 \pm 0,33 sur Kprakpra tandis qu'aucune progéniture n'a été enregistrée sur Portchahabim (Tableau 2). L'analyse de variance (Tableau 2) a révélé une différence significative entre les progénitures de $D$. porcellus sur les différents cultivars d'igname $(d l=3 ; \quad F=12,56$; $P=0,0021)$. Le test de séparation multiple de Student-Newman-Keuls (SNK) a révélé que l'effectif de la progéniture de $D$. porcellus a été significativement plus élevé sur le cultivar Otoukpanan que sur les autres cultivars (Singor, Kprakpra et Portchahabim) qui n'ont présenté aucune différence significative entre eux.

Les effectifs totaux de $D$. porcellus (œufs + larves + parents) retrouvés sur les différents cultivars ont été de 43,00 \pm 11,24 sur Otoukpanan, 8,00 \pm 3,51 sur Singor, 5,67 $\pm 2,3$ sur Kprakpra et 3,67 $\pm 0,33$ sur Portchahabim (Tableau 2). L'analyse de variance a révélé une différence significative entre les effectifs totaux de $D$. porcellus retrouvés sur les différents cultivars $(d l=3$; $F=11,70 ; P=0,0027)$. Le test de séparation multiple de Student-Newman-Keuls (SNK) a révélé que l'effectif total de $D$. porcellus retrouvé a été significativement plus élevé sur le cultivar Otoukpanan que sur les autres cultivars (c'est-à dire Singor, Kprakpra et Portchahabim) sur lesquels ces effectifs n'étaient pas significativement différents (Tableau 2). 
Tableau 1: Durées moyennes (en jours) des stades de développement de D. porcellus sur les cossettes préparées à partir des différents cultivars d'ignames testés.

\begin{tabular}{lccccc}
\hline Variétés & Euf & Larve & Nymphe & Totale & Longévité \\
\hline Kprakpra & $5,00 \pm 0,45 \mathrm{a}$ & $73,00 \pm 6,51 \mathrm{a}$ & $10,83 \pm 6,25 \mathrm{a}$ & $88,83 \pm 7,73 \mathrm{a}$ & $69,17 \pm 12,68 \mathrm{a}$ \\
Otounkpanan & $\mathbf{4 , 0 0} \pm \mathbf{0 , 2 4 a}$ & $\mathbf{5 2 , 3 5} \pm \mathbf{2 , 1 3 b}$ & $\mathbf{4 , 5 9} \pm \mathbf{0 , 4 5 a}$ & $\mathbf{6 0 , 9 4} \pm \mathbf{2 , 2 0 b}$ & $\mathbf{3 7 , 5 9} \pm \mathbf{4 , 5 8 a}$ \\
Portchahabim & $5,38 \pm 0,53 \mathrm{a}$ & $75,23 \pm 2,27 \mathrm{a}$ & $4,30 \pm 0,40 \mathrm{a}$ & $84,92 \pm 2,16 \mathrm{a}$ & $38,54 \pm 4,80 \mathrm{a}$ \\
Singor & $4,60 \pm 0,50 \mathrm{a}$ & $63,80 \pm 4,05 \mathrm{a}$ & $6,40 \pm 1,69 \mathrm{a}$ & $74,80 \pm 5,20 \mathrm{a}$ & $43,80 \pm 6,97 \mathrm{a}$ \\
\hline
\end{tabular}

NB: Dans une même colonne les valeurs affectées de la même lettre ne sont pas significativement différentes selon le test de Student-Newman-Keuls pour le paramètre considéré.

Tableau 2: Pertes moyennes de poids des cossettes (en \% du poids initial), nombre d'insectesparents vivants, de progéniture et nombre total d'insectes retrouvés sur les cossettes des différents cultivars d'igname.

\begin{tabular}{lcccc}
\hline Variétés & $\begin{array}{c}\text { Poids perdu } \\
(\%)\end{array}$ & $\begin{array}{c}\text { D. porcellus vivants } \\
\text { (sur 10) }\end{array}$ & Progéniture & $\begin{array}{c}\text { Nombre total de } \boldsymbol{D} \text {. } \\
\text { porcellus }\end{array}$ \\
\hline Otoukpanan & $\mathbf{2 , 0 0 \pm 0 0 a}$ & $\mathbf{8 , 0 0} \pm \mathbf{0 , 5 7 a}$ & $\mathbf{3 5 , 0 0} \pm \mathbf{1 1 , 1 3 a}$ & $\mathbf{4 3 , 0 0} \pm \mathbf{1 1 , 2 4 a}$ \\
Singor & $1,33 \pm 0,7 \mathrm{a}$ & $4,33 \pm 0,33 \mathrm{a}$ & $3,67 \pm 3,67 \mathrm{~b}$ & $8,00 \pm 3,51 \mathrm{~b}$ \\
Portchahabim & $1,33 \pm 0,7 \mathrm{a}$ & $3,67 \pm 0,33 \mathrm{a}$ & $0,00 \pm 0,00 \mathrm{~b}$ & $3,67 \pm 0,33 \mathrm{~b}$ \\
Kprakpra & $2,0 \pm 1,2 \mathrm{a}$ & $5,33 \pm 2,02 \mathrm{a}$ & $0,33 \pm 0,33 \mathrm{~b}$ & $5,67 \pm 2,3 \mathrm{~b}$ \\
\hline
\end{tabular}

NB: Dans une même colonne les valeurs affectées de la même lettre ne sont pas significativement différentes selon le test de Student-Newman-Keuls pour le paramètre considéré.

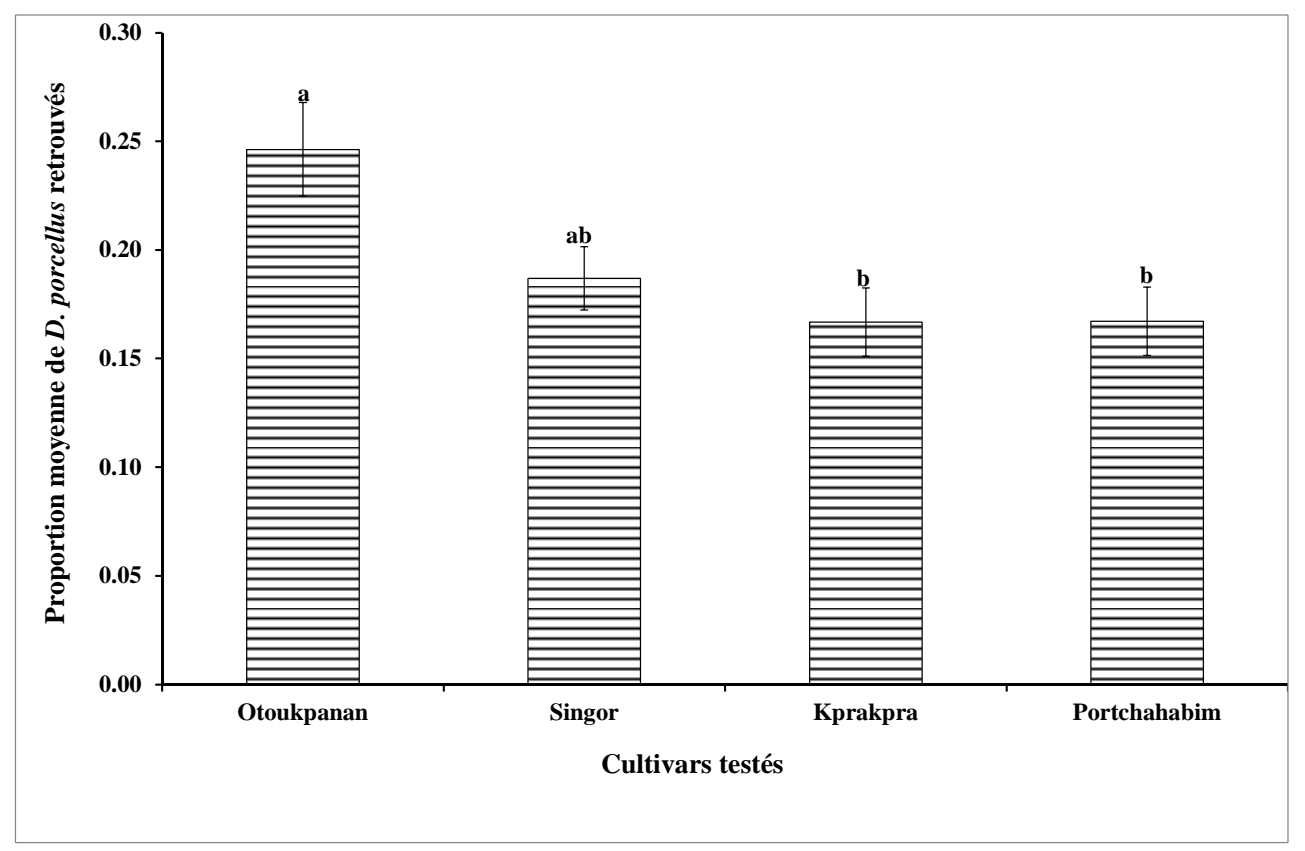


Figure 1: Distribution de D. porcellus vers les cossettes préparées à partir des 4 différents cultivars d'igname. Les barres surmontées d'une même lettre ne sont pas statistiquement différentes au seuil de 5\% selon le test de Student-Newman-Keuls (SNK).

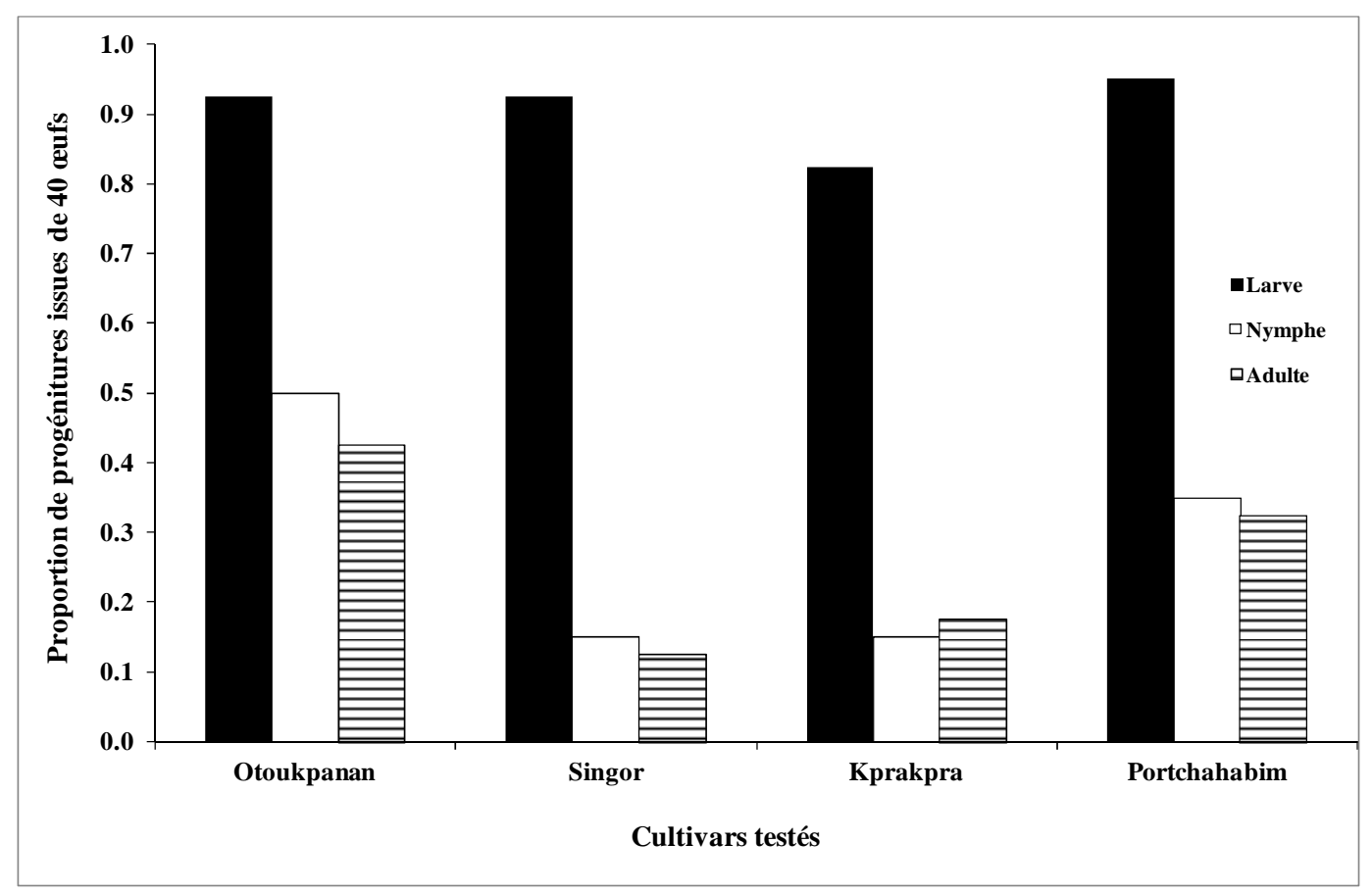

Figure 2: Proportion des progénitures issues de 40 œufs de D. porcellus sur les différents cultivars d'igname utilisées pour préparer les cossettes.

\section{DISCUSSION}

Cette étude, la première réalisée au Bénin (à notre connaissance), est l'une des rares à s'intéresser à la résistance à $D$. porcellus des variétés d'igname utilisées pour fabriquer les cossettes d'igname. Elle explore de façon non-exhaustive les mécanismes par lesquels certaines variétés d'igname échappent aux fortes infestations de $D$. porcellus, un redoutable ravageur des cossettes d'igname en stock. Cette étude vise également la classification de ces quatre variétés d'igname testées de point de vue de leur résistance relative aux attaques de ce ravageur. De toute évidence, la présente étude vient renchérir les acquis antérieurs sur la biologie du ravageur (Oni et Omoniyi, 2012), et sur ses préférences alimentaires, conditions sine qua non pour une lutte efficace contre ce redoutable ravageur.

En effet, nos résultats révèlent, dans un premier temps, qu'en conditions de choix au laboratoire, D. porcellus colonise des cossettes issues de certaines variétés d'igname et laisse d'autres. En effet, à l'issue de plusieurs tests de choix, il s'est avéré que les cossettes préparées avec la variété Otoukpanan ont été les plus envahies par le ravageur. Ainsi, 24,62 $\pm 2,17 \%$ des insectes testés se sont orientés vers les cossettes préparées avec cette variété tandis que seulement $16,68 \pm 1,58 \%$ ont été retrouvés sur les cossettes de la variété Kprakpra (Figure 1). On peut en déduire donc que le ravageur apprécie mieux les cossettes préparées avec la variété Otoukpanan que celles préparées avec la variété Kprakpra, ou plutôt que les 
cossettes issues de la variété Otoukpanan attirent plus les individus de D. porcellus que les cossettes issues des autres variétés testées. Cette dissemblance dans le choix des cossettes par le ravageur suppose, en effet, une différence dans l'attraction exercée par les différentes cossettes sur le ravageur, et que le ravageur ait pu, rien qu'en se basant sur ses réponses olfactives aux stimuli produits par les cossettes, s'apercevoir si la variété en face est bonne ou pas pour sa propre survie et celle de sa progéniture. Bien que de telles réponses soient très peu connues ou rapportées en ce qui concerne les ravageurs des denrées stockées, elles sont bien connues chez les arthropodes herbivores et leurs plantes-hôtes. A titre d'exemple, Onzo et al. (2012), ont rapporté que l'acarien prédateur, Typhlodromalus aripo De Leon (Acari : Phytoseiidae), utilisé dans la lutte biologique contre l'acarien vert du manioc, Mononychellus tanajoa Bondar (Acari : Tetranychidae), préfère les variétés de manioc poilues aux variétés non poilues parce qu'entre autres, ces variétés poilues protègent mieux le prédateur contre les radiations ultraviolettes du soleil (Onzo et al., 2010). En effet, les végétaux produisent des composés non nutritifs nommés composés secondaires ou allélochimiques qui entrent dans le système de défense de la plante contre les arthropodes ravageurs, et qui repoussent souvent ceux-ci (Gershenzon et Croteau, 1991). Autrement dit, ces plantes émettent des substances volatiles (toxines, réducteurs de digestibilité), ou présentent une morphologie (pubescence, dureté de tissu, etc.), qui font fuir ou découragent les arthropodes ravageurs (Sabelis et al., 1999; Norton et al., 2001). Ainsi donc, les cossettes issues de certaines variétés d'igname pourraient bien émettre de telles substances volatiles qui repousseraient les D. porcellus tandis que d'autres les attireraient. Par conséquent, les cossettes sur lesquelles les individus de D. porcellus sont plus abondants seraient certainement issues de variétés émettrices de substances volatiles attractives pour l'insecte. Si tel est le cas, il s'avère donc que bien que séchées les cossettes d'igname continuent d'influencer $D$. porcellus à travers des caractéristiques inertes telles que la couleur, l'odeur ou la texture, qui pourraient être répulsives ou attractives à $D$. porcellus selon la variété d'igname utilisée pour la préparation des cossettes.

Dans un second temps, nos résultats révèlent que la variété d'igname utilisée pour préparer les cossettes influe sur la durée de développement de $D$. porcellus. Des quatre variétés sur lesquelles $D$. porcellus a été élevé, le taux d'émergence le plus élevé $(42,5 \%$; Figure 2) ainsi que la durée de développement la plus courte $(60,94 \pm 2,20$; Tableau 1) de l'insecte ont été observés sur les cossettes préparées avec Otoukpanan. Ces résultats en complément à ceux du premier test, montrent que l'attraction de D. porcellus pour les cossettes issues de la variété Otoukpanan n'est pas simplement le fait d'un hasard. En effet, vu le taux d'émergence et la durée de développement de $D$. porcellus sur Otoukpanan, il apparaît clairement que la densité de population du ravageur sur les cossettes issues de Otoukpanan serait beaucoup plus élevée que sur celles obtenues sur les cossettes issues des autres variétés. Otoukpanan serait donc, non seulement attractif, mais assure une meilleure croissance démographique au ravageur.

Plusieurs travaux sur les relations entre insectes et plantes-hôtes confirment que le développement des insectes sur une plante donnée dépend des conditions offertes par celle-ci pour son développement (qualité, quantité, ennemis naturels, caractéristiques physiques et chimiques). En effet, lors d'une étude sur le papillon de nuit, Dasyses rugosella Stainton (Lepidoptera : Tineidae) élevé sur différentes variétés d'igname, Ashamo (2005), a rapporté que la composition du substrat (teneur en alcaloïdes, acides aminés, cendres, eau, tannins ...) influe sur le développement des insectes. Des résultats similaires ont été également obtenus par plusieurs auteurs lors des études sur les facteurs qui influenceraient le développement des bruches sur les graines de légumineuse (Janzen, 1977 ; Credland et Wright, 1988 ; Lale et Mustapha, 2000). Bien que ces facteurs n'aient pas été investigués dans notre étude, les composantes sus-citées pourraient bien agir comme facteurs antinutritionnels ou 
comme poisons pour réduire l'alimentation et affecter conséquemment la croissance des $D$. porcellus. Ceci est d'autant plus plausible qu'il a été reconnu que les cossettes d'igname contiennent des substances anti-nutritionnelles (Adedire et Oni, 1998).

Aussi, faut-il retenir de la présente étude que l'ampleur des dégâts causés par $D$. porcellus lors du stockage des cossettes d'igname varie en fonction de la variété d'igname. En effet, les dégâts les plus importants ont été enregistrés encore sur la variété Otoukpanan, cette même variété se révélant être celle sur laquelle les insectes retrouvés vivants ainsi que leurs progénitures étaient significativement les plus nombreux. La variété sur laquelle moins d'insectes vivants et moins de progéniture ont été enregistrés a été la variété Portchahabim (Tableau 2).

De tout ce qui précède, sommes-nous tentés d'affirmer que les cossettes de la variété Otoukpanan seraient le réel habitat naturel de D. porcellus. En effet, en plus d'être la variété préférée du ravageur, le nombre d'individus ayant accompli le plus rapidement leur développement y était plus élevé, et les dégâts enregistrés plus significatifs que sur cette variété que sur les trois autres testées à savoir : Singor, Kprakpra et Portchahabim. Il se dégage donc des trois études que les cossettes de la variété Otoukpanan se sont révélées être les plus vulnérables aux attaques de $D$. porcellus. En conséquence, la transformation des ignames de la variété Otoukpanan en cossettes pour un stockage à long terme doit être déconseillée aux producteurs. Cependant, les cossettes provenant de cette variété pourraient bien être utilisées par les chercheurs dans l'élevage de masse de D. porcellus à des fins expérimentales, notamment pour la recherche des ennemis naturels de ce redoutable ravageur.

\section{REMERCIEMENTS}

Les auteurs remercient l'Institut International d'Agriculture Tropicale (station $\mathrm{du}$ Bénin) pour son soutien financier et matériel à la réalisation de ce travail. Nous témoignons aussi notre gratitude au Dr. B.
Datinon et à Feu Dr. I. Zannou, ainsi qu'à messieurs R. Houndafotche et P. Sovimi de IITA-Benin pour leur assistance technique.

\section{REFERENCES}

Aboudou R, Auriole L. 2006. Impacts de la croissance urbaine sur les filières agricoles en Afrique de l'Ouest : cas de l'igname à Parakou, Bénin, Ifeas, Lares, document de travail Ecocité $\mathrm{n}^{\circ} 13$, www.ecocite.org, $51 \mathrm{p}$.

Adedire CO, Oni MO. 1998. Effect of host species on oviposition and development of the yam beetle Dinoderus porcellus Lesne (Coleoptera: Bostrichidae). ESN Occational Publ., 31: 243-248.

Adedoyin OT, Ojuawo A, Adesiyun OO, Mark F, Anigilage EA. 2008. Poisoning due to the yam flour consumption in five families in Ilorin, Central Nigeria. West. Afr. J. Med., 27(1): 41-43.

Adeleke SI. 2009. Food poisoning due to yam flour consumption in Kano (Northwest) Nigeria. Online J. Health Allied Scs. 8(2):10. http://www.ojhas.org/issue30/ 2009-2-10.htm.

Adesuyi SA. 1967. A survey of insect pests on dried yam and an investigation of the biology of the important species of Republic Nigeria. Stored Product Research Inst. Tech., 24(2): 95-99.

Akissoé N, Cornet D, Vernier P. 2007. Effet du tranchage mécanique des cossettes d'igname sur la qualité finale. Atelier final du projet FAO TCP igname.

Ashamo MO. 2005. Development of the yam moth, Dasyses rugosella Stainton (Lepidoptera: Tineidae) in different species of yam. : J. Plant. Dis. Protect., 4: 398-404.

Ategbo E, Bricas N, Hounhouigan J, Mitchikpe E, Nkpenu K, Orkwor G, Vernier P. 1997. Le développement de la filière cossettes d'igname pour l'approvisionnement des villes au Nigeria, au Bénin et au Togo. Actes du séminaire international Cirad-InraOrstom-Coraf. 3-6 juin 1997, Montpellier, France, 339-341.

Ayodeji SP, Olabanji OM, Adeyeri MK. 2012. Design of a Process Plant for the 
Production of Poundo Yam. Internat. J. Engineering, 6(1): 10-24.

Babajide OM, Oyewole OA, Obadina OB. 2006. An assessment of the microbiological safety of dry yam (gbodo) processed in South West Nigerian. Afr. J. Biotechnol., 6: 157-161.

Babarinde SA, Sosina A, Oyeyiola EI. 2008. Susceptibility of selected crops in storage to Sitophilus zeamais Motschulsky in southwestern Nigeria. $J$. Plant. Prot. Res., 48(4): 541-550.

Baco MN, Tostain S, Mongbo RL, Dainou O, Agbangla C. 2004. Gestion dynamique de la diversité variétale des ignames cultivées (Dioscorea cayenensis-D. rotundata) dans la commune de Sinendé au nord Bénin. Plant Genet. Resour. Newsl., 139: 18-24.

Bricas N, Vernier P. 2000. Dossier : la transformation de l'igname. Bulletin du réseau TPA $\mathrm{N}^{\circ} 18$, pp 5-12. http://www.infotpa.org/fileadmin/bulleti n/bulletin18.pdf.

Credland PF, Wright AW. 1988. The effect of artificial substrates and host extracts on oviposition by Callosobruchus maculatus (F.) (Coleoptera: Bruchidae). J. Stored Prod. Res., 24: 157-164.

Dansi A, Vernier P, Marchand J-L. 2003. Les variétés d'ignames cultivées: Savoirfaire paysans au Bénin. Dioscorea cayenensis, Dioscorea rotundata. FAO, Rome, Italy, 5-38.

Degras L. 1998. L'igname (Dioscorea spp.) dans les sociétés des Amériques. In: L'igname, plante séculaire et culture d'avenir. Actes du séminaire international Cirad-Inra-Orstom-Coraf, 3-6 juin 1997, Berthaud J, Bricas N, Marchand J-L. CIRAD. Montpellier, France; 33-43.

Demont M, Houedjoklounon A, Hounhouigan J, Mahyao A, Orkwor G, Stessens J, Tollens E, Touré M. 2003. Etude comparative des systèmes de Commercialisation d'igname en Côted'Ivoire, au Bénin et au Nigéria. Working Paper, $\mathrm{n}^{\circ}$ 79, Département d'Economie Agricole et de
l'Environnement, Universiteit Leuven.

Katholieke

Djoï D, Monhouanou JD. 2003. Etude de marché national sur les racines et tubercules et produits dérives, (vol 1): Rapport Principal.

FAO. 2012. FAOSTAT Database. Food and Agriculture Organization, Roma, Italy. Available online at URL: www.fao.org

Isah MD, Ayertey JN, Boateng BA. 2009. Suitability of Dried Chips of Plantain, Cocoyam, Yam And Cassava For The Development of The Larger Grain Borer Prostephanus truncatus (Horn) (Coleoptera: Bostrichidae). Internat. J. Applied Sci., 3(4): 12-20.

Janzen DH. 1977. How southern cowpea weevil larvae (Bruchidae: Callosobruchus maculatus ) die on nonhost seeds. Ecology, 58: 921-927.

Kossou DK. 1987. Un nouveau déprédateur dans le système post-récolte de la République du Bénin: Prostephanus truncatus (Horn) (Coleoptera: Bostrichidae). Carref. Rech., 2: 1-11.

Lale NES, Mustapha A. 2000. Efficacy and acceptability of neem (Azadirachta indica A. Juss) seed oil and pirimiphosmethyl applied in three storage devices for the control of Callosobruchus maculatus (F.) (Coleoptera: Bruchidae). Z. Pflanzenkrankh. Pflanzenschutz., 107(4): 399-405.

Loko YL, Dansi A, Tamo M, Bokonon-Ganta AH, Assogba P, Dansi M, Vodouhè R, Akoegninou A, Sanni A. 2013. Storage insects on yam chips and their traditional management in Northern Benin. The Scientific World Journal, 11p. doi:10.1155/2013/484536.

Norton AP, English-Loeb GM, Belden E. 2001. Host plant manipulation of natural enemies: leaf domatia protect beneficial mites from insect predators. Oecologia, 126: $535-542$.

Olajumoke OL, Agiang MA, Mbeh E. 2012. Proximate and anti-nutrient composition of white Guinea yam (Dioscorea rotundata) diets consumed in Ibarapa, South West region of Nigeria. J. Nat. Prod. Plant Resour., 2(2): 256-260. 
Oluwole OB, Awonorin SO, Henshaw F, Elemo GN, Ebuehi OAT. 2013. Assessment of Microbial Changes and Nutritional Qualities of Extruded White Yam (Dioscorea rotundata) and Bambara Groundnut (Vigna subterranea) Blends. Food Nutr. Sci., 4: 100-107.

Ondo OP, Kevers C, Dommes J. 2009. Plant Molecular Biology and Biotechnology Unit Sart Tilman, B 22 University of Liege, B-4000 Liege, Belgium.

Oni MO. 1995. Studies on the developmental biology of the dried yam bettle Dinoderus porcellus Lesne (Coleoptera: Bostrichidae). Unpublished M. Tech Thesis, Federal University of Technology, Akure Nigeria.

Oni MO, Omoniyi AO. 2012. Studies on Temperature Influence on Oviposition and Development of Immature Stages of the Yam Beetle Dinoderus porcellus Lesne (Coleoptera, Bostrichidae) on Dried Yam Species. J. Agr. Sci., 4(2): 213.

Onzo A, Hanna R, Sabelis MW. 2012. The predatory mite Typhlodromalus aripo prefers green-mite induced plant odours from pubescent cassava varieties. Exp. Appl. Acarol., 58: 359-370.

Onzo A, Sabelis MW, Hanna R. 2010. Effects of Ultraviolet Radiation on Predatory Mites and the Role of Refuges in Plant Structures. Environ. Entomol., 39: 695-701.

Osuji FNC. 1980. Observation on the beetles on dried yams and yam flour from three
Nigerian markets. Trop. Stored Prod. Res. Inst., 39: 35-38.

Sabelis MW, Janssen A, Bruin J, Bakker FM, Drukker B, Scutareanu P, van Rijn PCJ. 1999. Interactions between arthropod predators and plants: A conspiracy against herbivorous arthropods? In Ecology and Evolution of the Acari, Bruin J, van der Geest LPS, Sabelis MW (eds). Kluwer Academic Publishers: The Netherlands; 207-229.

SAS Institute. 2008. Software Version 9.2 (TSMO) Cary. North Carolina: USA.

Schäfer K, Goergen G, Borgemeister C. 2000. An illustrated identification key to four different species of adult Dinoderus (Coleoptera: Bostrichidae), commonly attacking dried cassava chips in West Africa. J. Stored Prod. Res., 36: 245252.

Vernier P, Dumont R. 1995. La production et l'utilisation de cossettes d'igname (Dioscorea cayenensis-rotundata) au Bénin: situation actuelle et perspective. Proc. 6th Symp. ISTRC-AB, 418-423.

Vernier P, Goergen G, Dossou RA, Letourmy P, Chaume J. 2005. Utilization of biological insecticides for the protection of stored yam chips. Outlook Agr., 34(3): 173-179.

Vernier P, Hounhouigan J, Bricas N. 2000. La transformation des ignames en cossettes et les préparations culinaires dérivées : une technique de transformation qui renforce la sécurité alimentaire en réduisant les pertes après-récolte et qui contribue à augmenter le revenu des femmes rurales. Eschborn : GTZ, 28 p. 